\title{
The effect of calibration and detector temperature on the reconstructed cone beam computed tomography image quality: a study for the workflow of the iCAT Classic equipment
}

\author{
Mark Plachtovics, DMD, ${ }^{\mathrm{a}}$ Janos Goczan, DMD, ${ }^{\mathrm{b}}$ and Katalin Nagy, DDS, $\mathrm{PhD}^{\mathrm{c}}$ \\ Objective. The image quality of the reconstructed dental cone beam computed tomography (CBCT) acquisition is strongly \\ dependent on the characteristics of the applied detector. Some flat panel detector (FPD) types require a warming-up period \\ (WUP) to achieve a steady-state temperature; and some of them, those that have a cesium iodide detector, may require a \\ calibration process (CP) after each WUP. \\ Study Design. Quality-assurance phantom was used for the evaluation of image quality, including spatial accuracy and \\ density response with and without WUP and CP using iCAT Classic equipment. \\ Results. The correlation between the measured gray values and the multidetector computed tomography (MDCT) values \\ deviated slightly from linearity (defined by the 45-degree line). There was no detectable difference in the spatial accuracy of \\ the four different scanning modes. \\ Conclusions. Although the WUP is important to reach the required steady-state temperature, the CP has a greater effect on the \\ image quality. (Oral Surg Oral Med Oral Pathol Oral Radiol 2015; a:1-8)
}

The cone beam computed tomography (CBCT) imaging process consists of several steps (Figure 1). The quality of the image may be influenced by a number of effects during the signal conversion chain. One of these is the optimal or nonoptimal state of the detector. For example, some instruments that use a certain type of flat panel detector (FPD) may require a warming-up period (WUP) for the detector to reach its steady-state temperature. Also, in certain cases, a calibration process $(\mathrm{CP})$ of the FPD is recommended for obtaining the ideal image quality. This requirement may escape the attention of some technicians. Also, during an emergency in the oral surgery department, an acquisition may have to be taken immediately after starting the equipment. Consequently, it is of utmost importance to investigate the impact of the WUP and the CP of the FPDs on image quality; this paper will focus on this aspect. For instance, for the iCAT Classic equipment (Imaging Sciences International, Hatfield, PA), the WUP is about 2 hours from the cold state. ${ }^{1}$ After the WUP, an electric fan prevents overheating and maintains a steady-state temperature of $30{ }^{\circ} \mathrm{C}$ in the FPD. The detector system is analyzed during the

\footnotetext{
${ }^{a}$ Research Associate, Faculty of Dentistry, University of Szeged, Tisza Lajos krt 64, Szeged, 6720, Hungary.

${ }^{\mathrm{b}}$ Research Associate, Resolution Specialist Treatment Centre, Becket House, Hendford, Yeovil, BA20 1 TE, UK.

${ }^{\mathrm{c}}$ Professor, Faculty of Dentistry, University of Szeged, Tisza Lajos krt 64, Szeged, 6720, Hungary

Received for publication Aug 30, 2014; returned for revision Dec 2, 2014; accepted for publication Dec 9, 2014.

(C) 2015 Elsevier Inc. All rights reserved.

$2212-4403 / \$$ - see front matter

http://dx.doi.org/10.1016/j.000o.2014.12.009
}

$\mathrm{CP}$, and suitable correction data are derived and later used during subsequent image acquisition to compensate for known deviations from the ideal behavior of the detector (Figure 1). These modified data would serve as the database for the reconstructed volume of the image. $^{2}$ Consequently, for quality assessment, instead of judging the quality of the image projected on the detector, it is more practical to examine the quality of the reconstructed image. Eventually, it is this image that the clinician would examine, not the one directly put out by the detector. Assessment of image quality is best achieved by following the quality assurance protocol for the equipment.

The objective in the present in vitro study is to investigate the measured gray values and the spatial accuracy of a quality-assurance phantom on the reconstructed image, which is recommended by the manufacturer for quality assessment of the equipment. Figure 2 shows the applied setup of the qualityassurance phantom in the iCAT Classic dental CBCT equipment used.

\section{MATERIALS AND METHODS}

To investigate the effect of the detector temperature and that of the calibration process of the FPD on CBCT

\section{Statement of Clinical Relevance}

The clinical relevance of our work is that it would help clinicians obtain better density accuracies in their cone beam computer tomography reconstructed acquisitions. 


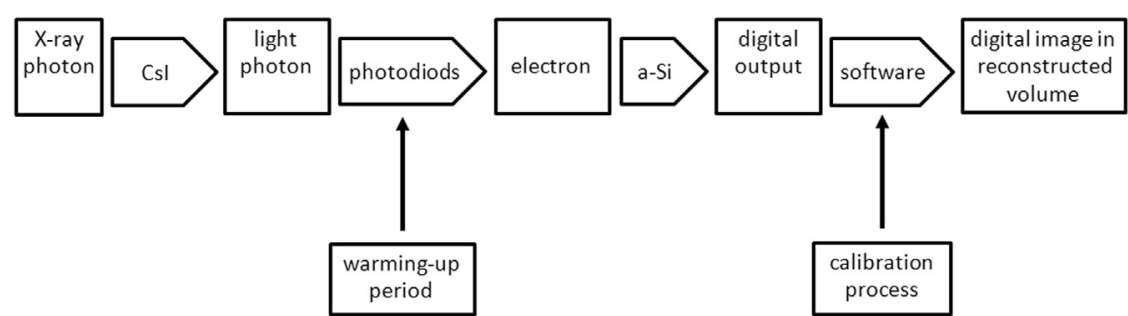

Fig. 1. Schematic figure of the signal conversion chain from $x$-ray to three-dimensional reconstructed image of the dental CBCT equipment, which incorporates an amorphous silicon image detector with a cesium iodide conversion layer. $C B C T$, cone beam computed tomography; $a$-Si, amorphous silicon; $C s I$, cesium iodide.

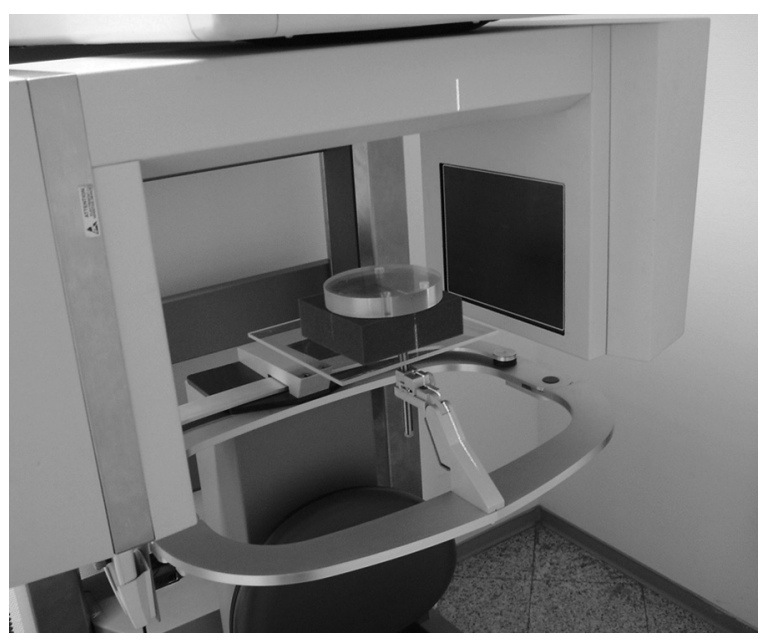

Fig. 2. Photograph of the configuration setup of the cylindrical (150-mm diameter) standard Imaging Sciences International (ISI) quality assurance CBCT phantoms in the iCAT Classic equipment. $C B C T$, cone beam computed tomography.

image quality, four different experimental conditions were used (Table I). Table II shows the technical parameters of these applied iCAT Classic CBCT scans. The iCAT Classic CBCT equipment is powered by the XoranCAT technology acquisition program, version 3.1.62 (Xoran Technologies Inc., Ann Arbor, MI). According to the operator's manual, ${ }^{1}$ all items were to be removed before calibrating the sensor, out of the field of view (FOV), and therefore these scans were taken without the head holder and chin rest of the instrument. The quality-assurance CBCT phantom was mounted horizontally, always in the same position in the center of the FOV on a foam layer (Figure 2). This Imaging Sciences International (ISI) phantom on the CBCT axial image is shown in Figure 3 and Figure 4. The image and location of the four insets are shown in Figure 3, and the appearance of the bar-pattern is shown in Figure 4. The quality assurance procedure would require two different measurements.
Table I. List and abbreviations of the four experimental conditions used for the flat panel detector (FPD) in the present study

\begin{tabular}{lc}
\hline Cold detector without calibration: & Cold detector with calibration: \\
COLD & COLD $+\mathrm{C}$ \\
Warm detector without & Warm detector with calibration: \\
calibration: WARM & WARM $+\mathrm{C}$ \\
\hline
\end{tabular}

$C O L D$, the acquisition was taken without warming-up period and calibration process of the FPD; $C O L D+C$, the abbreviation for the scan taken directly after the start of the equipment with cold detector but after the calibration process; WARM, indicates that the machine was on for at least 2 hours before the scan but no detector calibration was performed; WARM $+C$, scan protocol is consistent with the cone beam computed tomography scan with the complete calibration sequence after the warming-up period - the 2-hour warming-up period preceded the calibration process.

Table II. Performance characteristics for the applied acquisition mode of the iCAT Classic 14-bit gray-scale resolution CBCT dental imaging system*

\begin{tabular}{lc}
\hline Diameter of the FOV $(\mathrm{mm})$ & 154 \\
Height of the FOV $(\mathrm{mm})$ & 60 \\
Slice thickness of the reconstruction $(\mathrm{mm})$ & 0.2 \\
Reconstructed image matrix size (pixel) & $800 \times 800$ \\
Scan times (seconds) & 40 \\
Degrees of rotation & 360 degrees \\
Detector pixel height & 0.2540 \\
Tube current (mAs) & 36.12 \\
Tube voltage $(\mathrm{kVp})$ & 120 \\
\hline
\end{tabular}

$C B C T$, cone beam computed tomography; FOV, field of view.

*This acquisition mode is abbreviated as Maxl $6 \mathrm{~cm}, 40 \mathrm{Sec}, 0.2$ Voxel MaxRes.

First, the measured gray values in Hounsfield units were compared for the four different experimental conditions of the four homogenous density materials ${ }^{3,4}$ of the quality assurance CBCT phantom: Air $\left(1.29 \mathrm{~kg} / \mathrm{m}^{3}=\right.$ $\left.0.00129 \mathrm{~g} / \mathrm{cm}^{3}\right)$, low-density polyethylene (LDPE) $(0.92$ $\left.\mathrm{g} / \mathrm{cm}^{3}\right)$, acryl $\left(1.18 \mathrm{~g} / \mathrm{cm}^{3}\right)$ and Teflon $\left(2.16 \mathrm{~g} / \mathrm{cm}^{3}\right)$. On the basis of our previous results, ${ }^{4}$ no significant statistical variation was expected in this experiment when more scans were taken about the phantom with the same detector characteristics. For this reason, only one 

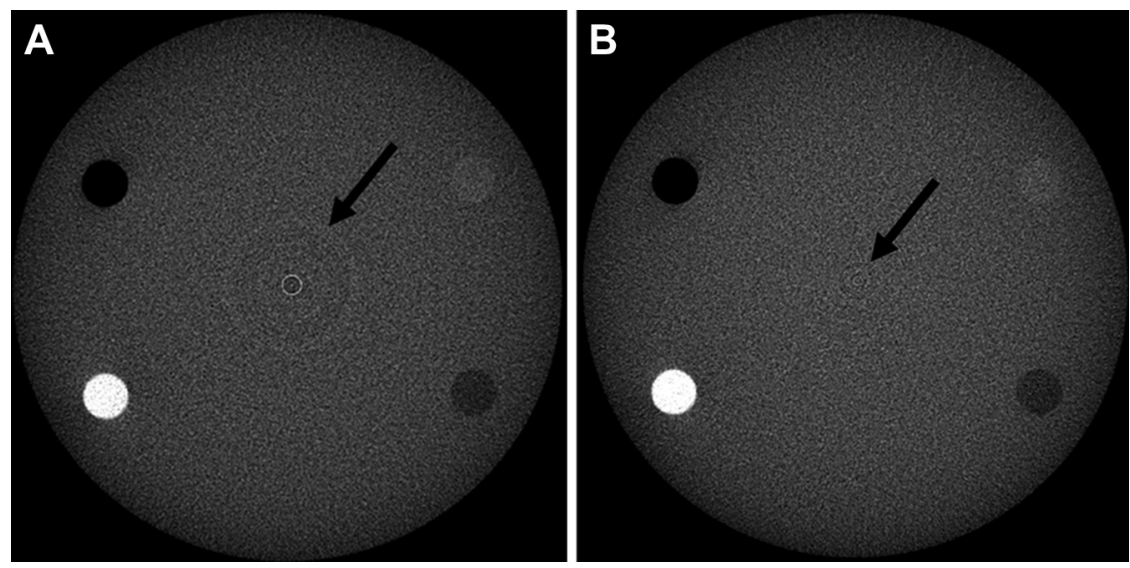

Fig. 3. Typical examples for ring artifacts in CBCT image which may result from failures of the calibration process (CP). Reconstructed images of the $150-\mathrm{mm}$ diameter standard ISI quality assurance phantom, which is made of air $\left(1.29 \mathrm{~kg} / \mathrm{m}^{3}=\right.$ $\left.0.00129 \mathrm{~g} / \mathrm{cm}^{3}\right)$, LDPE $\left(0.92 \mathrm{~g} / \mathrm{cm}^{3}\right)$, acryl $\left(1.18 \mathrm{~g} / \mathrm{cm}^{3}\right)$ and Teflon $\left(2.16 \mathrm{~g} / \mathrm{cm}^{3}\right)$ embedded in a Plexiglas cylinder. In the height of these two axial slices, the bar pattern phantom is not visible. On both of these acquisitions, ring artifacts are detectable (arrows) because these scans were taken without the $\mathrm{CP}$ of the detector. A, COLD. B, WARM. Properties for these experimental conditions of the applied acquisitions chosen for this study are shown in Table I. The four inserts are arranged in the following sequence: upper left hand side, air; lower left hand side, Teflon; lower right hand side, LDPE; upper right hand side, acryl. (Window 3000, level 605, applied filtering: "Sharpen $3 \times 3$ " by XoranCAT technology acquisition program, version 3.1.62 [Xoran Technologies Inc., Ann Arbor, MI]). CBCT, cone beam computed tomography; LDPE, low-density polyethylene.

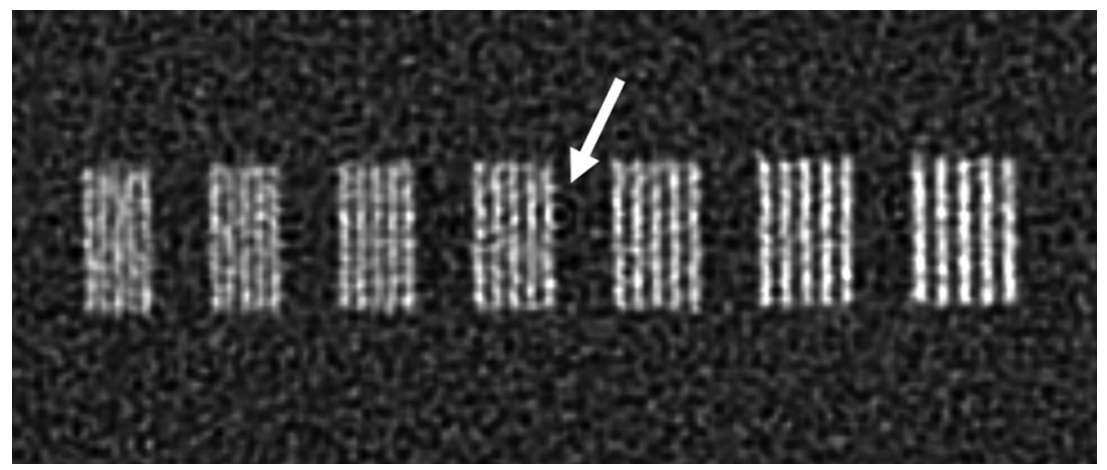

Fig. 4. Spatial resolution in the axial slice by cold detector without the CP (COLD). There were no visible differences in the appearance of the bar pattern phantom in the four applied scan conditions (Table I). The only detectable discrepancy from the other three experiential scan protocols, this ring artifact on the center of the axial views (arrow), was by the COLD operation mode. For better visibility, a slightly different setting was used: Window 3000, level 605, applied filtering: "Sharpen $3 \times 3$ ” by XoranCAT technology acquisition program, version 3.1.62.

experiment was performed using 10 different slice measurements that were properly averaged. The reconstructed volume was exported into the iCATVision software program, version 1.6.2.0. (Imaging Sciences International, Hatfield, PA). The gray values of the four 12-mm diameter uniformdensity cylinders (air, LDPE, acryl, and Teflon) were analyzed within a $60.2-\mathrm{mm}^{2}$ square-type region of interest. Ten different slices in the axial planes were investigated along the axial axis from the upper part of the phantom toward the lower region. To avoid distortion of the measured gray values, these analyzed axial slices were always chosen in such a way that they were not situated within the level of the "line par center" or the cone beam artefact. ${ }^{5}$ The average of these 10 values was calculated, and the standard deviation (SD) was determined. These average values of the four inserts were correlated with the multidetector computed tomography (MDCT) measured values (General Electric Medical Systems LightSpeed VFX Ultra, Tokyo, Japan, 120 kilovolt peak, $144.91 \mathrm{mAs}$ ) as described previously. ${ }^{4}$ The MDCT value is the expected CT number measured for the same ISI quality assurance phantom (Table III). ${ }^{4}$

Second, for checking the spatial accuracy of the CBCT images in the four applied scan conditions, the 
Table III. The expected CT numbers in Hounsfield units of four different phantom materials

\begin{tabular}{lr}
\hline & \multicolumn{1}{c}{ MDCT } \\
\hline Air & -999.24 \\
LDPE & -104.10 \\
Acryl & 115.16 \\
Teflon & 965.94 \\
\hline
\end{tabular}

$C T$, computed tomography; $M D C T$, multidetector computed tomography; $L D P E$, low-density polyethylene.

From Plachtovics M, Bujtar P, Nagy K, Mommaerts MY. Highquality image acquisition by double exposure overlap in cone beam computed tomography. Oral Surg Oral Med Oral Pathol Oral Radiol. 2014;117:760-767.

visibility of the bar pattern at the center of the ISI quality assurance phantom was investigated. These axial images, at the center of the height in the phantom, were analyzed by the XoranCAT technology acquisition program at the maximum magnification. The "Angio_Sharpen_high $5 \times 5$ " image filter was applied, and the set of the window level was 400/1000, as mentioned in the iCAT Classic operator's manual. ${ }^{1}$ These images from all four different applied experimental modalities were investigated on the same monitor (EIZO FlexScan L887 $51 \mathrm{~cm}$ (20.1 inch) class color LCD monitor, EIZO NANAO Corp., Matto, Japan).

\section{RESULTS}

Small changes in the gray values of the four inserts of the quality assurance phantom can be detected on the reconstructed CBCT image with the use or the omission of the appropriate $\mathrm{CP}$ and with alteration of the detector temperature. The measured gray values of the four inserts within the ISI quality assurance Plexiglas phantom are listed in Table IV. The averages and their SDs are shown in the last two lines of the same table (Table IV). The four experimental conditions resulted in four different patterns, as shown in the four curves in Figure 5, in which the $\mathrm{x}$-axis represents the expected $\mathrm{CT}$ numbers (in $\mathrm{HU}$ ), as summarized in Table III. It should be emphasized that in the case of WARM $+\mathrm{C}$ condition the deviation from the expected $\mathrm{CT}$ numbers (in $\mathrm{HU}$ ) and the measured gray values (in $\mathrm{HU}$ ) differ the least in the case of air and Teflon material, of which the latter is of similar density to cortical bone (Table V). In contrast to the above, in the case of inserts equivalent to the density of soft tissue, such as LDPE, and the inserts equivalent to the density of spongious bone, such as acryl, the cold sensor gave more accurate results but only with calibration $(\mathrm{COLD}+\mathrm{C})$. Air, the single insert material, showed more accurate mean values $(\mathrm{WARM}+\mathrm{C}, \mathrm{WARM}$ and COLD $+\mathrm{C}$ ) than MDCT value $(-999.24 \mathrm{HU})$. Furthermore, each of the 10

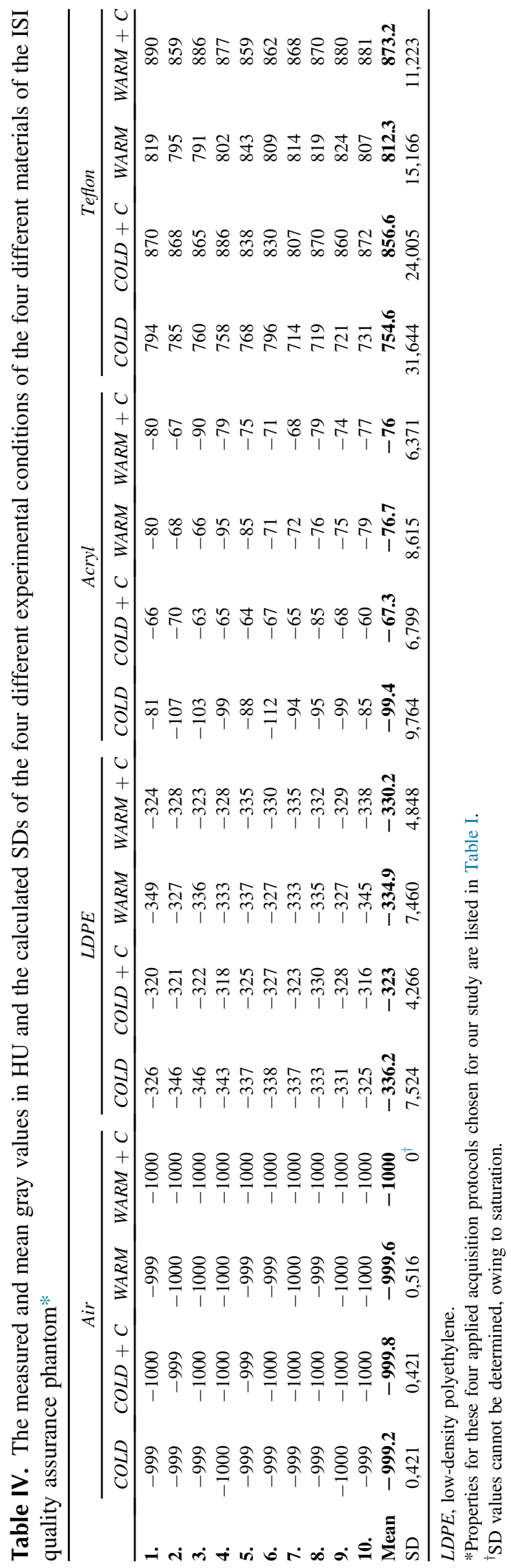




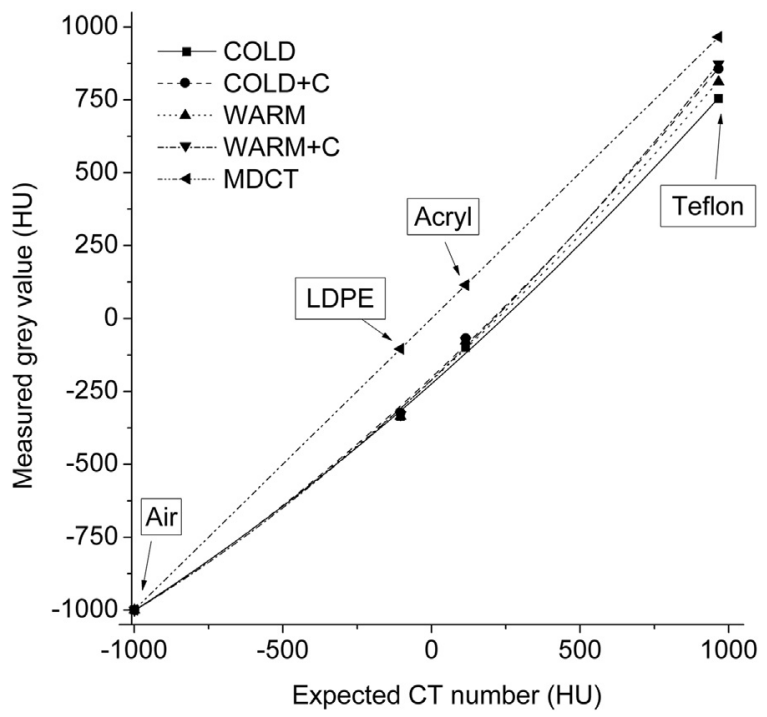

Fig. 5. The 45-degree straight line corresponds to perfect correlation, which is $\mathrm{Y}=\mathrm{X}$, where both variables are the multidetector computed tomography (MDCT) values (expected CT number in HU). Fitted quadratic equations of the four inserts of the quality assurance phantom are for the four experimental conditions of the acquisition protocol. The measured gray values correlated with the MDCT expected HU values slightly parabolically, and in the case of Teflon almost reached the perfect correlation (defined by the 45-degree line). Fitting parameters are summarized in Table VI. The measured data are listed in Table V. The expected CT numbers in HU are given in Table III. LDPE, low-density polyethylene; $M D C T$, multidetector computed tomography; $H U$, Hounsfield units.

measured gray values for the air insert were uniform $(-1000 \mathrm{HU})$ in the case of the applied WARM $+\mathrm{C}$ experimental condition.

During analysis of the spatial accuracy in the CBCT images of the four applied scan conditions, no difference was observed in visibility at the line par center. There was only one curious discrepancy in COLD operation mode. This involved projection of the concentric circles of ring artefacts on the bar pattern phantom, but it did not influence the resolution of the image (Figure 4).

\section{DISCUSSION}

During the past several years, the literature has included many reports in which different types of CBCT equipment were compared, ${ }^{6-11}$ with interesting results. However, some rather important characteristics of these instruments might have escaped the focus of scientific interest so far. The differences in the performances of the instruments described in this paper are related to the workflow of the acquisition procedure. Routine dental radiography, used more and more frequently, is increasing the radiation exposure of patients. The use of
Table V. Mean gray values (Y)*

\begin{tabular}{lrcrcr}
\hline & COLD & COLD $+C$ & WARM & WARM $+C$ & MDCT $^{4}$ \\
\hline Air & -999.2 & -999.8 & -999.6 & -1000 & -999.24 \\
Acryl & -99.4 & -67.3 & -76.7 & -76 & 115.16 \\
LDPE & -336.2 & -323 & -334.9 & -330.2 & -104.10 \\
Teflon & 754.6 & 856.6 & 812.3 & 873.2 & 965.94 \\
\hline
\end{tabular}

Properties for applied acquisition protocols chosen for this study are in Table I.

$M D C T$, multidetector computed tomography; LDPE, low-density polyethylene.

*Quadratic regression was used to establish the relationship between the measured gray values $(\mathrm{Y})$ and expected computed tomography (CT) numbers (Table III) of the four inserts: $\mathrm{Y}=\mathrm{AX}+\mathrm{BX}^{2}+\mathrm{C}$, where both $\mathrm{X}$ and $\mathrm{Y}$ are measured in Hounsfield units.

From Plachtovics M, Bujtar P, Nagy K, Mommaerts MY. Highquality image acquisition by double exposure overlap in cone beam computed tomography. Oral Surg Oral Med Oral Pathol Oral Radiol. 2014;117:760-767.

Table VI. Coefficients (A, B, and C) of the fitted quadratic equation for the four experimental conditions of the acquisition protocol

\begin{tabular}{lccc}
\hline \multicolumn{1}{c}{ Code } & $A$ & $B$ & $C$ \\
\hline COLD & 0.899 & $1.212 \times 10^{-4}$ & -224.33 \\
COLD + C & 0.952 & $1.519 \times 10^{-4}$ & -202.37 \\
WARM & 0.930 & $1.395 \times 10^{-4}$ & -212.76 \\
WARM + C & 0.961 & $1.691 \times 10^{-4}$ & -210.56 \\
\hline
\end{tabular}

Quadratic regression was used to establish the relationship between the measured gray values $(\mathrm{Y})$ and expected computed tomography (CT) numbers $(\mathrm{X})$ of the four inserts: $\mathrm{Y}=\mathrm{AX}+\mathrm{BX}^{2}+\mathrm{C}$, where both $\mathrm{X}$ and $\mathrm{Y}$ measured in Hounsfield units.

Properties for applied acquisition protocols chosen for this study are in Table I.

CBCT imaging has resulted in higher doses of radiation compared with other traditional dental radiographic methods. ${ }^{12-15}$ The overall amount of radiation doses to patients has increased. In accordance with the as-lowas-reasonably-achievable (ALARA) principle, the radiation dose for dental patients should be optimized to achieve the lowest practical level to meet the requirements of a specific clinical situation. ${ }^{14}$ Consequently, operators who are using CBCT units with operator-adjustable exposure settings should understand that these parameters affect both image quality and radiation dose; therefore, careful selection is required to follow the ALARA principle. ${ }^{2,14}$ For this reason, it is important that the best image quality be obtained at a certain radiation level. Molteni ${ }^{16}$ pointed out the importance of the application of imagespecific calibration of the equipment. However, in the case of certain types of equipment, the use of this extra step in the procedure could also result in less-thanperfect gray values. In accordance with the Journal of the American Dental Association (JADA) statement, ${ }^{14}$ 
it was possible in the present study to apply the quality control program recommended by the manufacturer. "Staffs of facilities using CBCT should establish a quality control program. This program can be based on the manufacturer's recommendations or can be established, implemented, and monitored by a qualified expert." 14

The present work aimed to assess image quality by examining the reconstructed volume. This has been achieved by the use of the bar pattern phantom and of the four inserts (air, LDPE, acryl, and Teflon) of the manufacturer's quality assurance phantoms.

The iCAT Classic equipment has a flat panel, which incorporates an amorphous silicon image detector with a cesium iodide conversion layer. This is a part of a complete digital x-ray imaging system (PaxScan 2520 Digital Imaging System, produced by Varian Medical Systems Inc., Salt Lake City, UT), which consists of the PaxScan the 2520 receptor, a command processor, and the power supply. This iCAT Classic CBCT instrument is claimed to work between 10 and $35^{\circ} \mathrm{C}$. ${ }^{1}$ The cesium iodide solid-state amorphous silicon FPD is an indirect, two-step x-ray conversion system. ${ }^{2,17,18}$ This two-step detection is based on the electronic signal generation from x-ray photons, which are not absorbed by the target. The first step is the conversion of the x-ray to visible light. When $\mathrm{x}$-ray photons strike the scintillator screen of the FPD, visible photons are emitted from the incorporated cesium iodide substrate. In the next step of the signal conversation chain, this visible light is converted by the pin photodiodes to electric charge, ${ }^{17,19}$ which is collected by the integrating amplifier and converted to output signal voltage, leading to digital data (Figure 1). ${ }^{18}$ The resistance characteristic of each of the photodiodes of the FPD depends on the temperature; this is why it is important to reach the steady-state temperature of the FPD during the WUP. The other important factor is the calibration process. Consequently, homogenous x-ray illumination, without any objects interfering with this radiation, is best for testing the detector and identifying pixel defects that show different gray shades. This CP is performed in four steps: (1) analysis of the detector panel; (2) offset calibration; (3) gain calibration; (4) high-gain calibration.

Two types of iCAT Classic equipment operation require WUP. One is a 5-second-long WUP of the x-ray tube, which is needed to ensure homogeneous radiation during gain and high-gain calibration. These last two steps of the $\mathrm{CP}$ always start with this 5-second exposure without $\mathrm{x}$-ray detection to ensure the warming up of the $\mathrm{x}$-ray tube. ${ }^{1}$ Following this, gain and high-gain calibrations are performed with homogeneous radiation, which is necessary to achieve uniform illumination to every pixel of the FPD.
The second type of operation requiring WUP of the iCAT Classic equipment is the WUP of the FDP, which lasts for 2 hours. This process has been described in some detail in this paper. The output electric charge of the photodiodes in the FDP can fluctuate even in the case of uniform photon density. This fixed pattern noise would affect the density response quality of the FPD. Although the $\mathrm{x}$-ray radiation is homogeneous and the FDP has reached the steady-state temperature, some pixels of the FPD will still show different shades. These would appear as lighter or darker ring artefacts ${ }^{20}$ around the axis of the rotation on the reconstructed image. The ring shape is created by the rotating motion of the detector during acquisition (Figures 3 and 4).The steady-state temperature of the FDP is not enough to prevent such a variable density response. The abovementioned four-step CP is also required, as it guarantees the optimal dose response of FPD pixels. The information gathered in this way would be used during the reconstruction process to compensate the differences in photodiode output (Figure 1). ${ }^{2}$

Of the measurements from the four inserts, only the air and Teflon measurements supported the conclusion that both the WUP and the CP are essential to achieve ideal density response in the reconstructed images. However, this is not contradictory, since dental CBCT technology can enable visualization of the high-contrast structure $^{17}$ in the acquisition, similar to MDCT for the "bone window" range. ${ }^{21}$ The numerical data of the Teflon insert indicate that the WUP and subsequently the $\mathrm{CP}$ are absolutely necessary for higher-density objects. According to the literature, ${ }^{5,17,21}$ CBCT technology is designed for relatively higher-density materials; therefore, lower-density materials such as fine trabecular bone, $\mathrm{D} 4{ }^{16}$ (corresponding to acryl), or soft tissues that are mimicked by LDPE cannot be studied quantitatively by this method while using this equipment. ${ }^{4,13}$

It is interesting to note that the four fitted quadratic curves of the four sets of measurements are below the ideal 45-degree line (Figure 5), which corresponds to the perfect correlation (Table V). However, if we were extrapolating the data to materials of higher density than Teflon, such as metallic dental implants, the quadratic curves would cross over the ideal 45-degree line. Note that the fitted function shown by Figure 5 corresponds very well to the MDCT value of air $(-999.24 \mathrm{HU})$ and is reasonably accurate for that of the Teflon (+965.94 HU). However, the deviation is the largest for LDPE and acryl (in the order of magnitude of $200 \mathrm{HU}),{ }^{4}$ which are near the mid-point $(0,0)$ of the fitted curve. In accordance with the above, the numerical data obtained for air and Teflon (Table IV) indicate that the CP is absolutely necessary. 
Returning to the fundamental question of accuracy, it has to be emphasized that the accuracy of this in vitro study was dependent on the selection of the 10 different slices that were investigated. The choices for the 10 different slices along the axial axis were very difficult and had to be made with great care. This extra care was necessary because either the presence within the same level of the cone beam artefact ${ }^{2,3,5,10,16,19}$ or that of the "line par center" $, 5,7$ would cause distortions of the gray values of the four inserts. Therefore, these slices were not considered appropriate for the density response examination.

The appearance of concentric circles in the reconstructed image of a well-functioning CBCT instrument is an indication that the CP with or without the WUP is incomplete. The appearance of these ring artefacts is the sign of pixel defects ${ }^{2,20}$ caused by the differences in the density responses of the pixels of the FPD. Therefore, it is beneficial to use homogeneous higher-density inserts that contain the quality assurance phantom, since an inhomogeneous structure, such as variably structured bones of inhomogeneous density, would cover the pixel defects.

\section{CONCLUSIONS}

The use of higher-density homogeneous insert materials, such as Teflon, in the quality assurance phantom proves the need for the WUP and subsequently the $\mathrm{CP}$ in certain types of FPD. The data obtained by the air insert has also supported this observation because the value $(-1000 \mathrm{HU})$, as set by the definition, was reached gradually during the COLD, WARM, $\mathrm{COLD}+\mathrm{C}$, and WARM $+\mathrm{C}$ experiment conditions. In contrast to this, homogeneous materials with lower density than Teflon, such as acryl and LDPE, do not mimic the density range of compact bone; therefore, they are not ideal to investigate the density response of this dental CBCT (Figure 5). Furthermore, it appears from the data obtained from the higherdensity objects that the effect of the WUP is noticeable, but the effect of the $\mathrm{CP}$ is more significant (Table IV). Consequently, both cold and warm detectors are useful, but only with appropriate calibration. From this, we may conclude that in the standardization of CBCT instruments that use the same or a similar type of FPD, both the WUP and the $\mathrm{CP}$ are important factors. However, the $\mathrm{CP}$ is considerably more important than the WUP for the FPD in preparing images of comparable quality with a fixed radiation dosage; therefore, these steps should be performed even in exceptional cases when the FPD has not had enough time to reach the steady-state temperature.

\section{REFERENCES}

1. iCAT Classic Operator's Manual. Hatfield, PA: Imaging Sciences International; 2010.

2. Scarfe WC, Farman AG. Cone-beam computed tomography: volume acquisition. In: Stuart CW, Michael JP, eds. Oral Radiology: Principles and Interpretation. St. Louis, MO: Mosby; 2014:185-198.

3. Bryant JA, Drage NA, Richmond S. Study of the scan uniformity from an iCAT cone beam computed tomography dental imaging system. Dentomaxillofac Radiol. 2008;37: 365-374.

4. Plachtovics M, Bujtar P, Nagy K, Mommaerts MY. High-quality image acquisition by double exposure overlap in cone beam computed tomography. Oral Surg Oral Med Oral Pathol Oral Radiol. 2014;117:760-767.

5. Pauwels R, Stamatakis H, Manousaridis G, et al. Development and applicability of a quality-control phantom for dental conebeam CT. J Appl Clin Med Phys. 2011;12:245-260.

6. Liang X, Jacobs R, Hassan B, et al. A comparative evaluation of cone beam computed tomography (CBCT) and multi-slice CT (MSCT) Part I. On subjective image quality. Eur J Radiol. 2010;75:265-269.

7. Pauwels R, Nackaerts O, Bellaiche N, et al. SEDENTEXCT Project Consortium. Variability of dental cone beam CT grey values for density estimations. $\mathrm{Br} J$ Radiol. 2013;86: 20120135.

8. Nemtoi A, Czink C, Haba D, Gahleitner A. Cone beam CT: a current overview of devices. Dentomaxillofac Radiol. 2013;42: 20120443.

9. De Vos W, Casselman J, Swennen GR. Cone-beam computerized tomography (CBCT) imaging of the oral and maxillofacial region: a systematic review of the literature. Int J Oral Maxillofac Surg. 2009;38:609-625.

10. Nackaerts O, Maes F, Yan H, Couto Souza P, Pauwels R, Jacobs R. Analysis of intensity variability in multislice and cone beam computed tomography. Clin Oral Implants Res. 2011;22: 873-879.

11. Oliveira ML, Tosoni GM, Lindsey DH, Mendoza K, Tetradis S, Mallya SM. Influence of anatomic location on CT numbers in cone beam computed tomography. Oral Surg Oral Med Oral Pathol Oral Radiol. 2013;115:558-564.

12. Horner K, Islam M, Flygare L, Tsiklakis K, Whaites E. Basic principles for use of dental cone beam computed tomography: consensus guidelines of the European Academy of Dental and Maxillofacial Radiology. Dentomaxillofac Radiol. 2009;38: 187-195.

13. Tyndall DA, Price JB, Tetradis S, Ganz SD, Hildebolt C, Scarfe WC. Position statement of the American Academy of Oral and Maxillofacial Radiology on selection criteria for the use of radiology in dental implantology with emphasis on cone beam computed tomography. Oral Surg Oral Med Oral Pathol Oral Radiol. 2012;113:817-826.

14. The American Dental Association Council on Scientific Affairs. The use of cone-beam computed tomography in dentistry. An advisory statement from the American Dental Association Council on Scientific Affairs. J Am Dent Assoc. 2012;143: 899-902.

15. The SEDENTEXCT Project. Radiation protection: cone beam CT for dental and maxillofacial radiology. Evidence based guidelines. Geneva, Switzerland: European Commission: 2011. Available at: http://wwwsedentexcteu/guidelines

16. Molteni R. Prospects and challenges of rendering tissue density in Hounsfield units for cone beam computed tomography. Oral Surg Oral Med Oral Pathol Oral Radiol. 2013;116:105-119. 
17. Miracle AC, Mukherji SK. Cone beam CT of the head and neck, part 1: physical principles. Am J Neuroradiol. 2009;30: 1088-1095.

18. Chotas HG, Dobbins JT, Ravin CE. Principles of digital radiography with large-area, electronically readable detectors: a review of the Basics. Radiology. 1999;210:595-599.

19. Kalender WA, Kyriakou Y. Flat-detector computed tomography (FD-CT). Eur Radiol. 2007;17:2767-2779.

20. Schulze R, Heil U, Groß D, et al. Artefacts in CBCT: a review. Dentomaxillofac Radiol. 2011;40:265-273.

21. Schulze D, Blessmann M, Pohlenz P, Wagner KW, Heiland M. Diagnostic criteria for the detection of mandibular osteomyelitis using cone-beam computed tomography. Dentomaxillofac Radiol. 2006;35:232-235

Reprint requests:

Mark Plachtovics, DMD

Faculty of Dentistry

University of Szeged

Tisza Lajos krt 64

Szeged, 6720

Hungary

Plachtovics.mark@stoma.szote.u-szeged.hu or placmark@gmail.com 